Check for updates

Cite this: Nanoscale Adv., 2019, 1, 1763

\title{
Nanoscale mechanical control of surface electrical properties of manganite films with magnetic nanoparticles $\dagger$
}

\author{
Borislav Vasić, (D) *a Zorica Konstantinović, (DD ${ }^{\mathrm{b}}$ Elisa Pannunzio-Miner,,$^{\mathrm{t}}$ \\ Sergio Valencia, (D) Radu Abrudan, (D) $\S^{\mathrm{e}}$ Radoš Gajića and Alberto Pomar (D)
}

Mechanical control of electrical properties in complex heterostructures, consisting of magnetic $\mathrm{FeO}_{x}$ nanoparticles on top of manganite films, is achieved using atomic force microscope (AFM) based methods. Under applied pressure of the AFM tip, drop of the electrical conductivity is observed inducing an electrically insulating state upon a critical normal load. Current and surface potential maps suggest that the switching process is mainly governed by the flexoelectric field induced the sample surface. The relaxation process of the electrical surface potential indicates that the diffusion of oxygen vacancies from the bulk of the manganite films towards the sample surface is the dominant relaxation mechanism. The magnetic $\mathrm{FeO}_{x}$ nanoparticles, staying attached to the sample surface after the rubbing, protect the underlying manganite films and provide stability of the observed resistive switching effect. The employed mechanical control gives a new freedom in the design of resistive switching devices since it does not depend on the film thickness, and biasing is not needed.

Received 22nd October 2018

Accepted 18th February 2019

DOI: 10.1039/c8na00301g

rsc.li/nanoscale-advances properties. In this context, one of the most studied mechanisms is resistive switching - an electrically induced change of the resistance of various thin metal-oxide films. ${ }^{1-7}$ This research is mainly driven by possible applications in new data storage devices such as resistive random access memories. ${ }^{4-7}$

In order to fully understand complex physicochemical processes during resistive switching, investigations at the nanoscale are of particular relevance, where methods based on atomic force microscopy (AFM) are prerequisite. ${ }^{8-11}$ At the same time, AFM could provide novel methods for the manipulation of resistive switching. Namely, in addition to the electrical control, strain engineering is a simple approach to tailor the electrical properties of metal oxide films. ${ }^{12-17}$ The significant coupling between the strain and the electrical properties is based on flexoelectricity (an internal electric field resulting from a strain gradient) and the inverse Vegard effect (changes of ion concentrations due to a stress). ${ }^{18}$ The flexoelectricity is typically small. However, it has recently been shown that stress and stress gradients at the nanoscale can lead to sizable effects. ${ }^{19-21}$

A large nanoscale gradient of stress can be obtained by using the tip of an AFM which has been employed for mechanically induced resistive switching ${ }^{22-29}$ and switching of ferroelectric polarization..$^{30-36}$ Since purely mechanical control is independent of film thickness and biasing is not needed, it could provide new prospects for the resistive switching. While recent studies ${ }^{22-29}$ have been focused on the mechanically induced switching of homogeneous metal-oxide films, it is interesting to investigate the phenomena in the presence of nano-objects on the film surface. In this respect, nanoparticles (NPs) constitute a model system as 
they not only improve mechanical properties, ${ }^{37}$ but also may provide enhanced functionalities such as novel magnetotransport phenomena $^{38}$ or optoelectronic capabilities, ${ }^{39,40}$ and they could serve as novel magnetic tunnel junctions ${ }^{\mathbf{4 1 , 4 2}}$ or memory devices. ${ }^{43}$

Here we investigate the local mechanical control of the surface electrical properties of a heterostructure consisting of a manganite film with magnetic $\mathrm{FeO}_{x}$ NPs. The AFM tip is used to generate a local stress by rubbing the sample surface, and the resulting changes in local conductivity and electrical surface potential are subsequently measured by conductive AFM (CAFM) and Kelvin probe force microscopy (KPFM), respectively. It is shown that for a high enough normal load, the manganite surface can be switched to an insulating state. At the same time, the sample exhibits weak room-temperature magnetism due to the presence of $\mathrm{FeO}_{x}$ NPs which stay well attached to the sample surface even after the rubbing at a high normal load.

\section{Experimental}

\subsection{Sample preparation}

Samples consisting of iron oxide $\mathrm{FeO}_{x}$ NPs assembled onto manganite films, were grown by radio frequency (RF) magnetron sputtering on the top of (001)-oriented $\mathrm{SrTiO}_{3}$ (STO) substrates. Two manganite families were considered with very different properties, fully spin polarized $\mathrm{La}_{0.67} \mathrm{Sr}_{0.33} \mathrm{MnO}_{3}$ (LSMO) on one side and insulating $\mathrm{La}_{0.5} \mathrm{Sr}_{0.5} \mathrm{Fe}_{0.5} \mathrm{Mn}_{0.5} \mathrm{O}_{3}$ (LSFMO) thin film on the other side. Nanostructured thin films were deposited under a pure oxygen partial pressure $(0.19 \mathrm{mbar})$ at a high temperature (850-900 ${ }^{\circ} \mathrm{C}$ ) with a wide range of nominal thickness (between $20 \mathrm{~nm}$ and $100 \mathrm{~nm}),{ }^{44}$ while the detailed microstructure of LSMO films can be also found in ref. 44. Iron oxide NPs have been deposited under a pure oxygen pressure of $0.06 \mathrm{mbar}$ at $700{ }^{\circ} \mathrm{C}$.

\subsection{Structural and magnetic characterization}

The surface morphology of nanostructured thin films was characterized by field emission scanning electron microscopy (SEM) (QUANTA FEI 200 FEG-ESEM). The crystal structure of manganite films was characterized by X-ray diffraction based reciprocal space mapping using a Bruker AXS GADDS system equipped with a 2D X-ray detector.

Magnetic properties were measured with a superconducting quantum interference device magnetometer (SQUID) (Quantum Design). The structural and magnetic nature of iron oxide NPs was studied by X-ray absorption spectroscopy (XAS) and X-ray magnetic circular dichroism (XMCD). The synchrotron experiments were performed at room temperature by using the ALICE chamber $^{45}$ at the PM3 beamline of the electron storage ring BESSY II of the Helmholtz-Zentrum Berlin. The radiation impinged on samples at a grazing angle of $30^{\circ}$. The polarization of the incoming radiation was set to circular $\left(P_{c}=0.92(3)\right)$. Data were acquired across the $\mathrm{Fe}_{3,2}$ edges by means of total electron yield. The XMCD spectrum was obtained for a fixed helicity of the incoming polarization by reversing the magnetization direction at every data point from positive to negative by means of an external magnetic field $( \pm 0.1 \mathrm{~T})$. Data were obtained in magnetic remanence. XMCD was defined as the difference in the absorption for the curves obtained after applying the external magnetic field $+0.1 \mathrm{~T}(\beta+)$ and $-0.1 \mathrm{~T}(\beta-)$, respectively.

\subsection{AFM measurements}

AFM measurements were performed using an NTEGRA Prima AFM system from NT-MDT under ambient conditions. Tapping AFM mode was used for the topographic imaging. Simultaneously, phase imaging was performed by recording the phase lag of the employed AFM cantilevers.

Electrical measurements were done using C-AFM and KPFM. In C-AFM, local electrical currents were measured in contact mode, while DC voltage was applied between the sample and the AFM tip. KPFM was employed to measure the contact potential difference (CPD) between the AFM tip and the sample surface. The CPD is equal to the difference in the work functions of the tip and sample. KPFM measurements were done using a standard two-pass technique. In the first pass, sample topography was measured in tapping mode. In the second pass, the AFM cantilever was lifted by $30 \mathrm{~nm}$ and the sum of AC and variable DC voltage was applied between the cantilever and the sample. In the second pass, the lifted probe, only electrically excited and with a switched-off mechanical feedback loop, followed the topography measured in the first pass. Then, the CPD in every point of a two-dimensional AFM image was the value of the variable DC voltage which canceled the electrically excited oscillations of the AFM cantilever in the second pass.

The procedure for the mechanical control of the surface electrical properties was the following: first, the local electrical current or CPD was measured on an area of $2 \times 2 \mu \mathrm{m}^{2}$, and then we switched to contact AFM mode and only the inner and central part of $1 \times 1 \mu^{2}$ was rubbed at an increased normal force (in the order of $1 \mu \mathrm{N}$ ), whereas after the rubbing, we switched back to C-AFM or KPFM mode, and measured either local electrical currents or electrical surface potentials, respectively, on the initial area of $2 \times 2 \mu \mathrm{m}^{2}$. Hereafter, the rubbing will stand for controllable scanning at increased normal load in order to induce local changes of the electrical properties.

Both AFM imaging, rubbing and electrical measurements were done using diamond coated and nitrogen doped DCP20 probes from NT-MDT. They have triangular cantilevers with the typical force constant of $48 \mathrm{~N} \mathrm{~m}^{-1}$ and a resonant frequency of $420 \mathrm{kHz}$. Diamond coatings make these probes wear resistive, while a high doping with nitrogen provides excellent conductivity. Therefore, these robust and conductive probes are suitable for the rubbing in contact AFM mode at high normal loads (in the order of $\mu \mathrm{N}$ ) and subsequent AFM imaging as well as electrical measurements many times. Since C-AFM measurements were done in contact mode as well, in order to avoid any influence of the applied normal force from the AFM tip during the C-AFM scanning, these measurements were done at low normal load from $50-100 \mathrm{nN}$.

\section{Results and discussion}

Manganites are an important class of metal oxides, especially in the context of resistive switching, because of both 
magnetoresistive ${ }^{\mathbf{4 6 , 4 7}}$ and electroresistive properties. ${ }^{\mathbf{4 8 - 5 0}}$ Strain engineering is an additional and simple approach to tailor their properties. $^{25,51-54}$ Epitaxial manganite films are usually elastically strained due to a lattice mismatch between the films and underlying substrates (STO in the considered case). This strain facilitates growth of various self-organized morphologies such as pit arrays ${ }^{55-57}$ which can serve as templates for subsequent self-assembly of NPs. ${ }^{\mathbf{4 4}}$ The strain state in our films is analyzed by the asymmetric reciprocal space mapping of the reflection (103) for the nanostructured $100 \mathrm{~nm}$ thick LSMO film grown on top of the STO substrate (Fig. S1(a) of the ESI $\dagger$ ). As observed previously, ${ }^{55}$ the LSMO film seems to be fully strained with the in-plane lattice constant close to $a_{\|, \mathrm{STO}}=3.905 \AA$. On the other hand, the estimated out of-plane lattice constant $a_{\perp, \text { LSMO }}=$ 3.882(6) $\AA$ is slightly smaller than the corresponding bulk value of $3.889 \AA$, as expected due to presence of a tensile strain. ${ }^{44,55}$ Nevertheless, the higher value of the out-of-plane parameter 3.882(6) A compared with the one of the fully strained films of $3.868 \AA$ (ref. 58) indicates the presence of a small quantity of oxygen vacancies, as previously discussed..$^{55}$

The AFM topographic and the corresponding phase image of the LSMO film with $\mathrm{FeO}_{x}$ NPs are given in Fig. 1(a) and (b), respectively. In the topographic image, NPs are visible as bright domains. They are better resolved in the phase image, where they are dark and with a pronounced contrast compared to the underlying LSMO film. The phase images recorded in forward and backward directions were the same, implying that the observed phase difference was due to the material contrast (not (a)

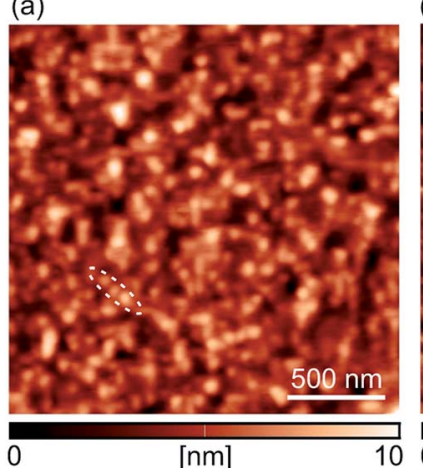

(c)

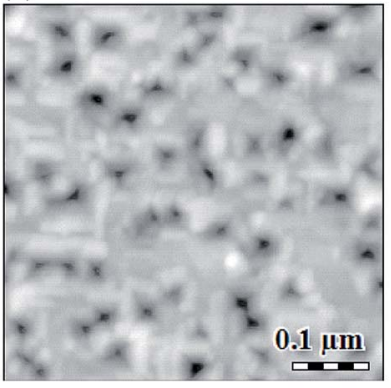

(b)

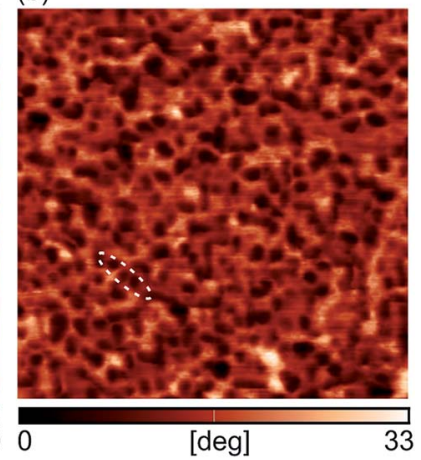

(d)

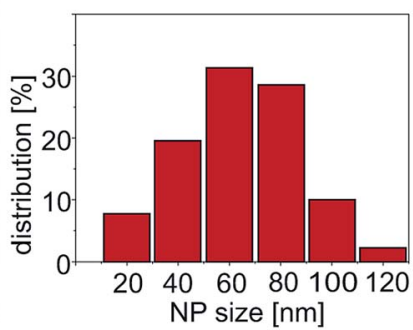

Fig. 1 Sample morphology: (a) AFM topographic image (z-scale is 10 $\mathrm{nm}$ ), and (b) AFM phase image acquired during the imaging in tapping mode. As an example, an array of three NPs is encircled in both the topographic and phase images. (c) SEM image and (d) the size distribution of iron-oxide NPs. just a topographic artifact) between NPs and the LSMO substrate. As a reference, an array of three NPs is marked by dashed lines in both topographic and phase images. The SEM image of the sample is depicted in Fig. 1(c). According to the SEM images, the surface coverage of the film by NPs was around $50 \%$, while the NP size distribution is presented in Fig. 1(d) showing that the average NP size is around $60 \mathrm{~nm}$.

The magnetic characterization reveals that iron-oxide NPs provide weak room-temperature magnetism as shown in Fig. S1(b) of the ESI. $\dagger$ At $300 \mathrm{~K}$, the magnetization disappears in bare manganite films and remains principally only in the structures with $\mathrm{FeO}_{x}$ NPs as depicted in Fig. S4 of the ESI. $\dagger$ According to the results of XAS and XMCD measurements, the NPs are mostly $\gamma-\mathrm{Fe}_{2} \mathrm{O}_{3}$ (the results of XAS and the fit of the XMCD curve are presented in Fig. S2 of the ESI $\dagger$ ).

The influence of the local pressure from the AFM tip on the electrical properties of the manganite film decorated with ironoxide NPs is presented in Fig. 2(a). The current maps were measured by C-AFM on six different $2 \times 2 \mu \mathrm{m}^{2}$ areas, after the rubbing of inner $1 \times 1 \mu^{2}$ domains with the AFM tip at increasing normal force, starting from $0.32 \mu \mathrm{N}$ to $1.6 \mu \mathrm{N}$. As can be seen, after the rubbing, the electrical current of inner square domains is decreased. At a normal load of $1.6 \mu \mathrm{N}$, the inner
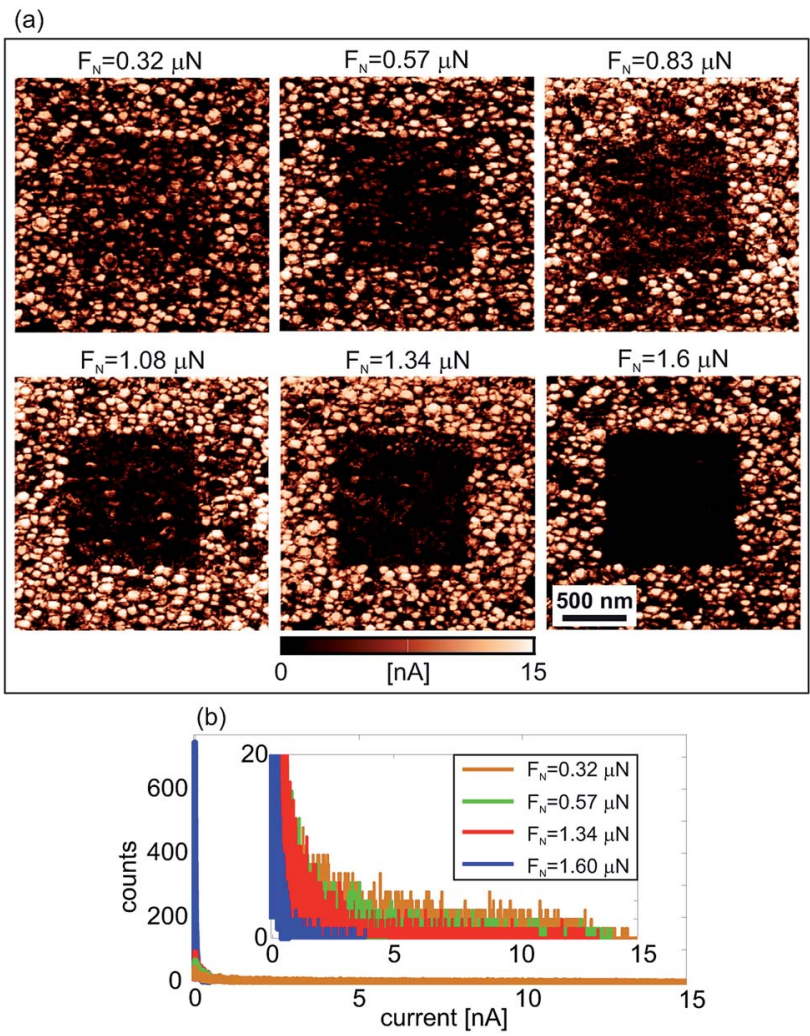

Fig. 2 (a) $2 \times 2 \mu \mathrm{m}^{2}$ current maps measured by C-AFM (at the sample bias voltage $U s=2 \mathrm{~V}$ ) after the rubbing of inner $1 \times 1 \mu \mathrm{m}^{2}$ domains at specified normal force (from $0.32 \mu \mathrm{N}$ to $1.6 \mu \mathrm{N}$ ). The results were obtained on six different sample regions. The current scale is $15 \mathrm{nA}$. (b) Histograms of the current distribution on rubbed domains in (a) for the selected normal forces. In the inset, the histograms are saturated at 20 counts for a better visibility. 
square is switched to a practically insulating state (with almost zero electrical current). The histograms of the current distribution measured only on rubbed domains are given in Fig. 2(b). All current peaks are located around zero, but they become much more pronounced with increasing normal load. For the highest load, almost all points in the histogram are located around zero (just few of them with non-zero current) showing that the sample surface is really switched to an insulating state.

Fig. 3(a) presents the CPD maps measured by KPFM on $2 \times 2$ $\mu \mathrm{m}^{2}$ areas after the rubbing of inner $1 \times 1 \mu \mathrm{m}^{2}$ regions with the AFM tip at an increasing normal force from $0.62 \mu \mathrm{N}$ to $2.4 \mu \mathrm{N}$. As in the previous case, the CPD maps were measured on different sample locations. As can be seen, CPD increases on the rubbed domains. Since the CPD stands for the difference between the work functions of the AFM tip and the sample, an increase in the CPD implies a lower work function of the sample. Therefore, the KPFM results indicate the existence of an electric field originating from negative charges on the sample surface.

The typical histogram of the CPD distribution is depicted in Fig. 3(b) for a normal load of $2.4 \mu \mathrm{N}$. There are two clearly resolved peaks corresponding to the rubbed (the peak at a higher CPD) and non-rubbed (the peak at a lower CPD)

(a)

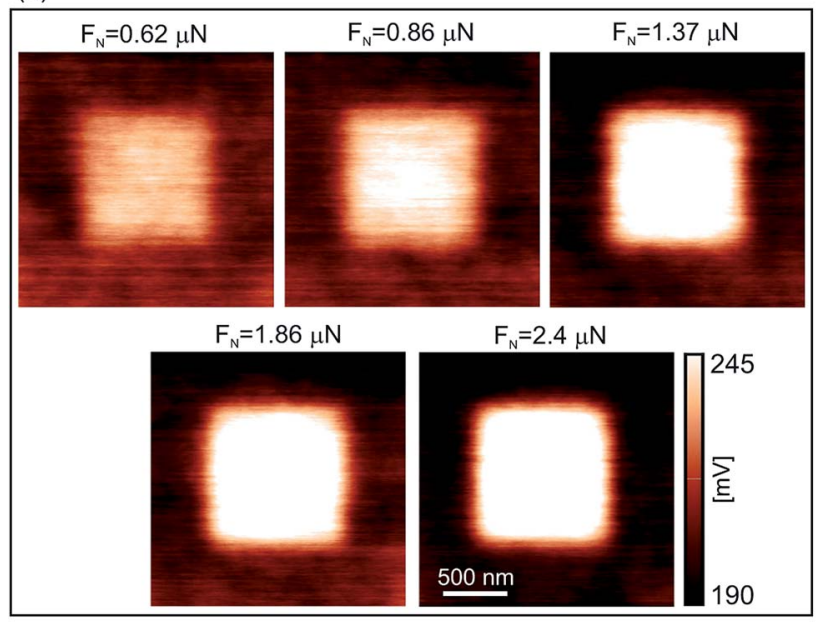

(b)
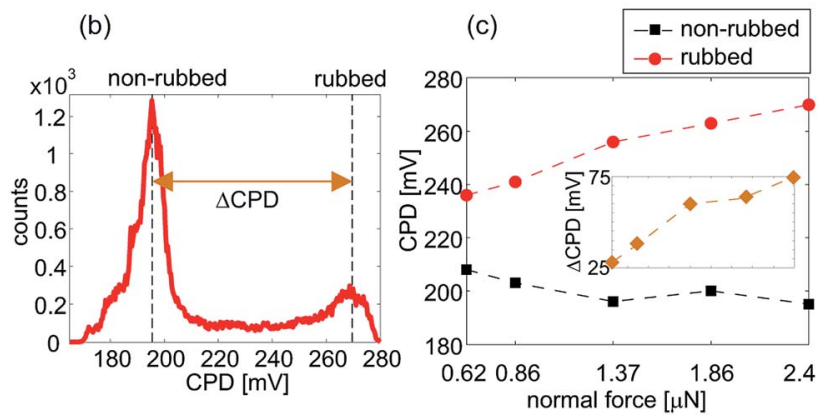

Fig. 3 (a) $2 \times 2 \mu \mathrm{m}^{2}$ CPD maps measured by KPFM after the rubbing of inner $1 \times 1 \mu \mathrm{m}^{2}$ domain at specified normal force (from $0.62 \mu \mathrm{N}$ to 2.4 $\mathrm{nN}$ ). The CPD scale is 190-245 mV. (b) Histogram of the CPD distribution for a normal load of $2.4 \mu \mathrm{N}$. (c) Changes in the CPD and $\triangle C P D$ on rubbed and non-rubbed regions in (a) as a function of the normal force. domains. Histograms were calculated for all CPD maps in this manner, whereas the histogram peaks were selected as CPD representatives as a function of a normal load. The corresponding results, together with a difference in the CPD between rubbed and non-rubbed domains ( $\triangle \mathrm{CPD}$ ), are given in Fig. 3(c). As can be seen, $\Delta$ CPD continuously increases with the normal load from around $25 \mathrm{mV}$ to $75 \mathrm{mV}$.

The topographic images of the sample surface before and after the rubbing (at a high normal force of $1.6 \mu \mathrm{N}$ ) are given in Fig. 4(a) and (b), respectively. The $1 \times 1 \mu \mathrm{m}^{2}$ rubbed domain is marked by a dashed square. The rubbed region is only slightly darker than the surrounding, non-rubbed part. The histograms and corresponding fits of the height distribution within the square domains before and after the rubbing are given in Fig. 4(c). As can be seen, the mean height is decreased by around $0.3 \mathrm{~nm}$ after the rubbing, meaning that the sample surface is locally compressed along the perpendicular direction. Similar results were obtained for the sample with the LSFMO film as depicted in the topographic images in Fig. S6(b) ESI, $\dagger$ again for the cases recorded before and after the rubbing (at a high normal force of $1.34 \mu \mathrm{N}$ ). These topographic images illustrate that NPs were not pushed away by the AFM tip during the rubbing even at very high normal loads. This was confirmed by the absence of accumulated NPs along the rims of the inner 1 $\times 1 \mu \mathrm{m}^{2}$ rubbed domains. Still, in addition to the small local compression of the sample surface within the rubbed domains, the topographic images after the rubbing show that the height
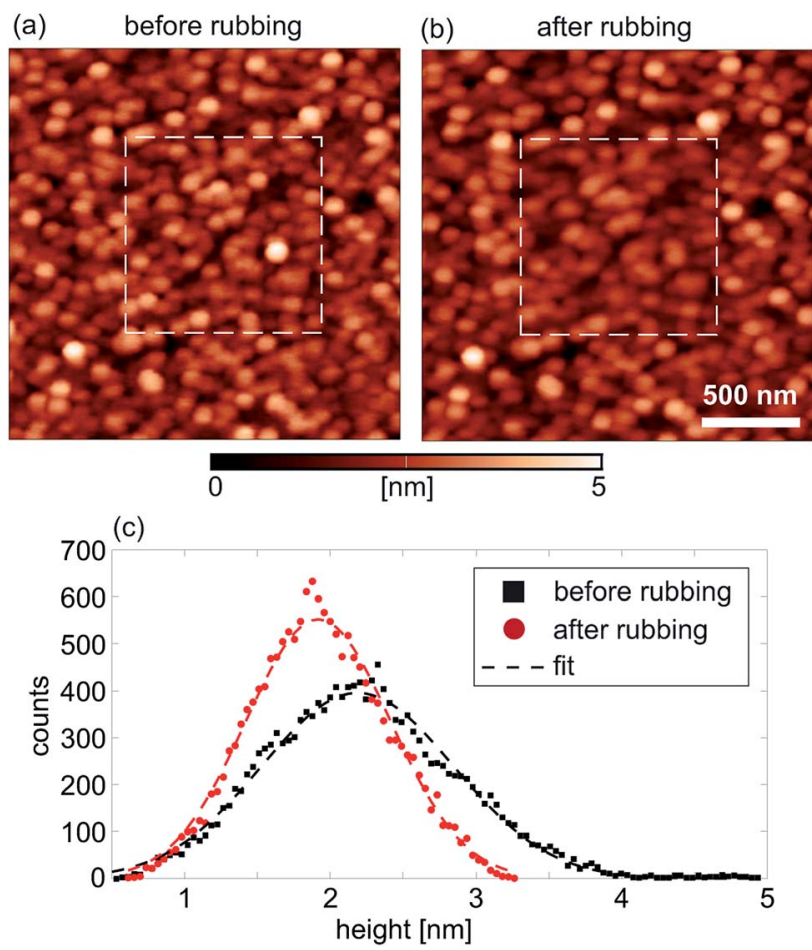

Fig. 4 AFM topographic images of the sample surface acquired (a) before and (b) after the rubbing of the inner $1 \times 1 \mu \mathrm{m}^{2}$ domain (marked by dashed lines) at a normal force of $1.6 \mu \mathrm{N}$. z-scale is $5 \mathrm{~nm}$. (c) Histogram of the height distribution within the square domains marked in parts (a) and (b). 
of some NPs is slightly decreased. This can be seen by lower brightness and clarity of these NPs. Therefore, NPs stay stable on the film surface and they are not removed, but some of them can be worn since their height is decreased due to local scratching. Still, the most important finding is that the NPs are tightly attached to the underlying film which stays protected.

The protection by NPs is two-fold. They mechanically protect the underlying manganite film from normal load applied by the AFM probe preventing any morphological damage of the film and facilitating a stable and reliable switching process. At the same time, NPs prevent aging of manganite films due to their exposure to the environment. Namely, it is well known that environmental factors deteriorate electrical properties (conductivity for example) of thin metal-oxides, usually due to detrimental reactions with various species from the atmosphere. This is the reason why higher conductivity was usually observed on areas of the manganite film covered by NPs, as can be seen in Fig. 2(a).

In order to further explain the observed effects, the rubbing with a grounded probe at increased normal load was combined with the scanning in contact mode using a biased AFM tip, but at a low normal force. CPD maps after such manipulations are depicted in Fig. 5(a) and (b). In Fig. 5(a), the $2 \times 2 \mu \mathrm{m}^{2}$ square domain was firstly rubbed with the grounded tip, and then the inner $1 \times 1 \mu^{2}$ domain was scanned in contact mode with a bias voltage of $U_{\mathrm{t}}=-1 \mathrm{~V}$ applied to the AFM tip. In Fig. 5(b), the order was the opposite, the larger square domain was scanned in contact mode at $U_{\mathrm{t}}=-1 \mathrm{~V}$, while the inner one was rubbed with the grounded tip. In both cases, the rubbing with the grounded tip and increased normal load leads to an increased CPD. This is exactly the opposite to the case with the applied negative voltage (in this case, the CPD was lowered). Therefore, the local pressure from the AFM probe has a similar

(a)

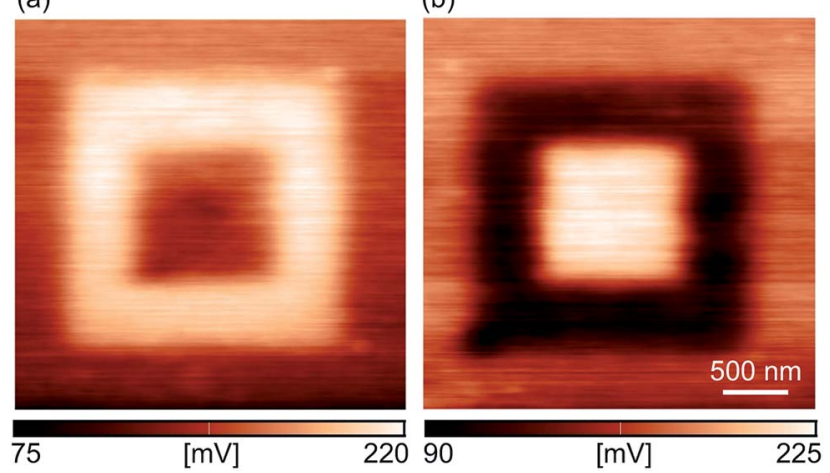

Fig. $53 \times 3 \mu \mathrm{m}^{2}$ CPD maps of the sample rubbed with both grounded (at high normal load) and biased (low normal load and negative bias voltage) tips: (a) first, the inner $2 \times 2 \mu \mathrm{m}^{2}$ domain was rubbed with the grounded AFM probe and at a normal force $0.48 \mu \mathrm{N}$, and then, the smaller inner $1 \times 1 \mu \mathrm{m}^{2}$ domain was scanned in the contact mode at a low normal force $0.16 \mu \mathrm{N}$ and with a tip bias voltage of $-1 \mathrm{~V}$, and (b) first, the inner $2 \times 2 \mu \mathrm{m}^{2}$ domain was scanned in the contact mode at a low normal force of $0.16 \mu \mathrm{N}$ and with a tip bias voltage of $-1 \mathrm{~V}$, and then the smaller inner $1 \times 1 \mu \mathrm{m}^{2}$ domain was rubbed with the grounded AFM probe and at a normal force of $0.48 \mu \mathrm{N}$. The CPD scale is (a) $75-220 \mathrm{mV}$ and (b) $90-225 \mathrm{mV}$. effect as the rubbing with a positive bias voltage applied to the AFM tip.

According to the type of conducting path, there are two resistive switching mechanisms: with a filamentary and an interface-type conducting path. ${ }^{4}$ The switching mechanism of LSMO films belongs to the second type and it is based on oxygen vacancy migrations. ${ }^{59}$ This mechanism is usually characterized by the formation of a Schottky barrier at the interface between a metal electrode and a semiconducting metal-oxide film., ${ }^{\mathbf{4 , 6 0}}$ The width and height of the barrier can be tuned by applying a bias voltage which controls the oxygen vacancy concentration within the charge depletion layer at the metal-semiconductor interface.

In the considered case of mechanically induced resistive switching, instead of an externally applied bias voltage, the width and height of the Schottky barrier between the AFM tip (DCP20 probes are highly conductive, so they can be considered as metallic ones) and sample surface are controlled by the inverse Vegard effect and flexoelectric field. According to the Vegard law of a chemical expansion, the local stress and strains are proportional to the mobile ion concentration. ${ }^{18}$ Generally, the unit cell volume increases with the oxygen vacancy concentration. ${ }^{26}$ In the considered case, the sample surface rubbed with the AFM tip is locally compressed along the surface normal, so the oxygen vacancy concentration is decreased. As a result, an excess of negative charges on the sample surface appears. This is in accordance with the results of KPFM measurements, where an increased CPD was observed on rubbed parts, meaning that the Fermi level was locally raised. The electrical transport in LSMO is based on the hopping of electrons between adjacent $\mathrm{Mn}^{3+}$ and $\mathrm{Mn}^{4+}$ ions across oxygen ions. ${ }^{61}$ During the rubbing, the oxygen ion concentration beneath the tip is increased (since the oxygen vacancy concentration is decreased), so this effect does not seem to be a mechanism for the observed decrease of the electrical conductivity.

At the same time, a local and non-uniform compression of a sample surface leads to a strain gradient and a local flexoelectric field. This field is oriented from the sample surface toward the bulk. ${ }^{31}$ This pushes oxygen vacancies away from the sample surface, while attracting negative charges toward the sample surface, again in accordance with the KPFM results. Since the C-AFM maps in Fig. 2(a) were measured with a positive bias voltage applied to the sample, the induced flexoelectric field is an obstacle for the electron transport from the AFM tip to the sample. Therefore, the induced flexoelectric field makes an additional potential barrier for the electron transport resulting in lower currents as observed in the C-AFM maps.

The inverse Vegard effect and flexoelectric field can be coupled as well. The LSMO films grown on the STO substrate are under in-plane tensile strain due to a lattice mismatch as can be seen from the asymmetric reciprocal space map given in Fig. S1(a) of the ESI. $\dagger$ This tensile strain can induce an in-built flexoelectric field. ${ }^{21}$ The strain can be relaxed by increasing the oxygen vacancy concentration. ${ }^{21}$ However, in a sample rubbed with the AFM tip, the oxygen vacancy concentration is further decreased due to the inverse Vegard effect. The strain is then 
even more enhanced, potentially leading to an additional increase of the flexoelectric field. As a result, both the inverse Vegard effect and flexoelectric field could raise the potential barrier supporting the observed changes of the electrical properties.

The efficiency and robustness of the resistive switching process were tested also in the case of an insulating LSFMO thin film with $\mathrm{FeO}_{x}$ NPs on top. At the same time, besides different conductivities of the underlying film (here the measured current on non-rubbed areas was up to $50 \mathrm{pA}$, that is, three order of magnitude lower than on the LSMO film with NPs), the surface coverage of the LSFMO film by NPs was increased in order to check its influence on the switching process. The asymmetric reciprocal space map and the in-plane magnetization curves for this sample are given in Figs. S3(a) and (b) of the ESI, $\uparrow$ respectively. The morphology of the investigated sample is depicted in Fig. S5 of the ESI. $\dagger$ The surface coverage of the LSFMO substrate by $\mathrm{FeO}_{x}$ NPs is more than $80 \%$. As a result, the AFM tip is practically in direct contact only with the NPs (on the other hand, due to a lower surface coverage of the LSMO film, there are parts with a bare LSMO substrate which can be directly probed with the AFM tip). The influence of a local pressure on the surface electrical properties is displayed in Fig. S6 (C-AFM analysis) and S7 (KPFM analysis) of the ESI. $\dagger$ They illustrate that the local electrical current drops, while the local CPD grows with the normal force applied during a rubbing. The CPD maps measured after the combined rubbing with a grounded tip at an increased normal load and a negatively biased tip are given in Fig. S8 of the ESI. $\uparrow$ The observed changes in the CPD indicate that the rubbing with the grounded tip is equivalent to the applying a positive bias voltage by the AFM tip.

According to the presented results, the electrical properties were changed in the same way for both samples despite a different surface coverage by NPs. We hence conclude that the observed changes are dominantly related to manganite films since the surface coverage by NPs does not have a significant influence. Therefore, during the rubbing with the AFM tip, the applied mechanical load is transferred across NPs to the underlying manganite films, so the AFM tip does not need to be in direct contact with the films. Since $\mathrm{FeO}_{x}$ NPs are not laterally connected, electrical currents go from the AFM tip across NPs to manganite films, or directly from the tip to the films. After that, charge carriers are transported laterally through the manganite films to an external electrical contact.

The results of KPFM measurements indicate that the rubbing with a grounded AFM tip and increased normal load induce localized charges on a sample surface. In metal-oxides generally, localized charges appear due to local changes in the oxygen vacancy concentration. In order to measure the time evolution of the localized charges, the CPD was measured as a function of a time. The resulting CPD maps after the rubbing of the inner $1 \times 1 \mu^{2}$ square domain are given in Fig. 6(a) (immediately after the rubbing, after $2 \mathrm{~h}$ and after $3 \mathrm{~h}$ ) and Fig. 6(b) (immediately after the rubbing, after $3 \mathrm{~h}$ and after $6 \mathrm{~h}$ ) for LSMO and LSFMO films, respectively.

The results for the time-dependent measurements are summarized in Fig. 7(a1) and (b1) for the samples with LSMO and LSFMO films, respectively, displaying the CPD for both rubbed and non-rubbed regions. The representative values of the CPD were calculated from the corresponding histograms, as in the previous analysis. The CPD on the rubbed regions was decreasing rather slowly. After around $4 \mathrm{~h}$ and $6 \mathrm{~h}$ for the LSMO and LSFMO films, respectively, the CPD was almost equal on both rubbed and non-rubbed domains. The difference in the CPD between two domains, $\triangle \mathrm{CPD}$ as a function of time is depicted in Fig. 7(a2) and (b2) for the LSMO and LSFMO films, respectively. The experimental results were fitted with the following curve: $\Delta \mathrm{CPD}(t)=\Delta \mathrm{CPD}(0) \exp (-t / \tau)$ (given by the dashed line), where $t$ is time in hours, while $\tau$ is the time constant of the relaxation process. For the sample with the LSMO film, $\tau=1.75 \mathrm{~h}$, while for that with the LSFMO film, the relaxation was much slower, with the time constant $\tau=3.5 \mathrm{~h}$.

The rate constant of the relaxation of oxygen vacancies can be calculated according to the measured CPD in the following way: ${ }^{62}$

$$
\gamma(t)=\frac{\Delta \mathrm{CPD}(t)-\Delta \mathrm{CPD}(0)}{\Delta \mathrm{CPD}(\infty)-\Delta \mathrm{CPD}(0)},
$$

where $\Delta \mathrm{CPD}(t)$ is the change in the CPD between rubbed and non-rubbed domains, while $\Delta \mathrm{CPD}(0)$ and $\triangle \mathrm{CPD}(\infty)$ stand for $\Delta \mathrm{CPD}$ at the initial moment and at the end of the relaxation, respectively. Generally, oxygen incorporation into a metal-oxide film can be divided into two processes, the surface reaction and diffusion in the sample bulk, ${ }^{6-64}$ so the rate constant can be deduced in the following way:

$$
\gamma(t)=\gamma_{\text {surf }}(t)+\gamma_{\text {diff }}(t)
$$
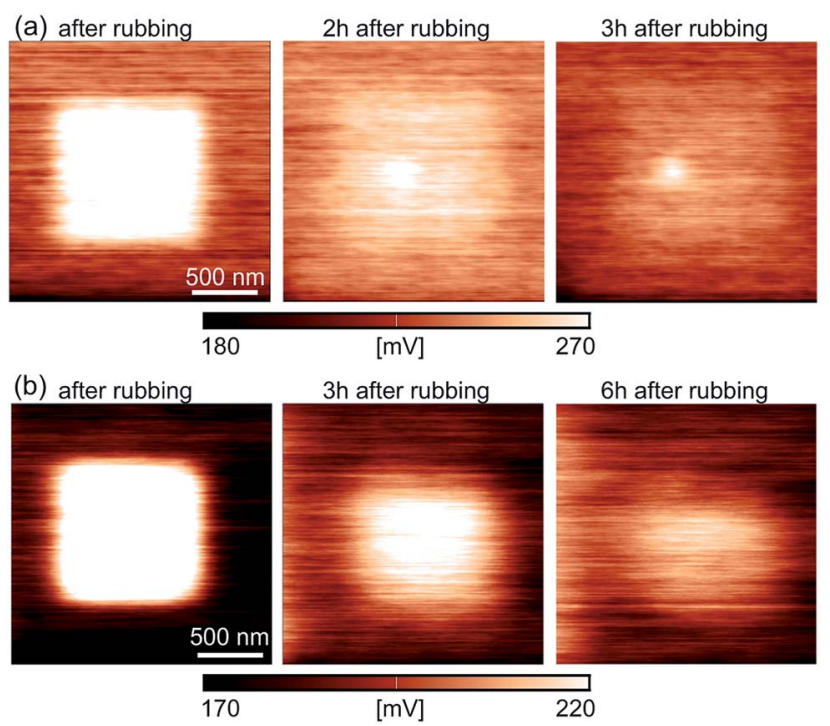

Fig. 6 Evolution of CPD maps after the rubbing (at a normal force of $0.55 \mu \mathrm{N}$ ) of the inner $1 \times 1 \mu \mathrm{m}^{2}$ domain as a function of time: (a) CPD maps of the sample with a LSMO substrate measured immediately after the rubbing, after $2 \mathrm{~h}$, and after $3 \mathrm{~h}$, (b) CPD maps of the sample with a LSFMO substrate measured immediately after the rubbing, after $3 \mathrm{~h}$, and after $6 \mathrm{~h}$. The CPD scale is (a) 180-270 $\mathrm{mV}$ and (b) 170$220 \mathrm{mV}$. 

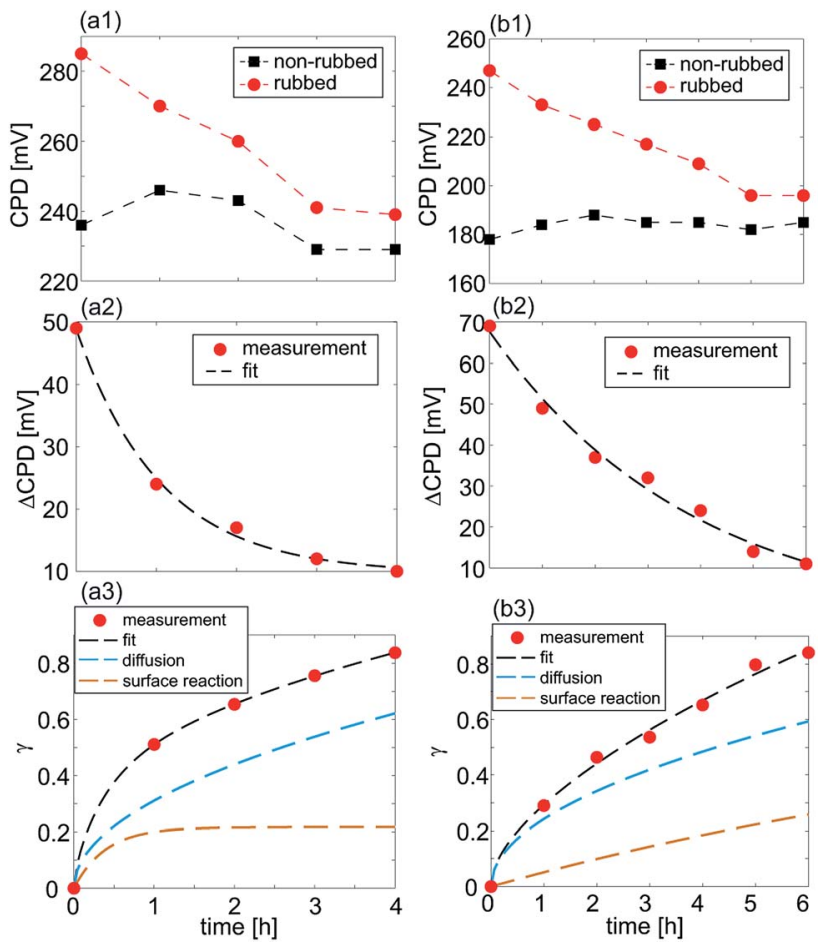

Fig. 7 Evolution of CPD, $\triangle \mathrm{CPD}$, and $\gamma$ as a function of time for the (a) LSMO and (b) LSFMO samples. Top row (a1) and (b1): CPD on rubbed and non-rubbed areas. Middle row (a2) and (b2): CPD difference, $\triangle C P D$, calculated as the difference between CPDs on rubbed and non-rubbed areas. Dashed lines stand for the fit to experimentally obtained points. Bottom row (a3) and (b3): factor $\gamma$ obtained from the experimental results (points) and the corresponding fit (dashed lines) with the individual contribution of $\gamma_{\text {surf }}(t)$ and $\gamma_{\text {diff }}(t)$.

where $\gamma_{\text {surf }}$ and $\gamma_{\text {diff }}$ stand for the rate constants of the surface reaction and diffusion, respectively. The surface reaction part can be approximated with an exponential law $\gamma_{\text {surf }}(t) \sim 1-$ $\exp \left(-k_{\mathrm{s}} t / L\right)$, where $k_{\mathrm{s}}$ is the surface reaction constant and $L$ is the film thickness. The diffusion part is proportional to $\gamma_{\text {diff }}(t) \sim \sqrt{t}$. The overall rate constant $\gamma(t)$ together with the contributions of $\gamma_{\text {surf }}(t)$ and $\gamma_{\text {diff }}(t)$ are given in Fig. $7($ a3 $)$ and (b3) for both samples, LSMO and LSFMO films, respectively. As can be seen, the diffusion has larger contribution in the overall relaxation of oxygen vacancies. Accordingly, the relaxation process can be described in the following way. The rubbing with the AFM tip leads to a local sample compression and a decrease of the oxygen vacancy concentration at the sample surface. When the AFM tip is removed, the sample surface relaxes slowly, with a time constant in the order of hours. During that time, oxygen vacancies diffuse from the sample bulk toward the surface in order to reach the initial concentration.

KPFM maps measured after the rubbing suggest that the mechanical interaction AFM tip-sample could induce trapped charges in the sample surface due to either contact charging (local pressure in a single point) or triboelectrification (during AFM tip motion in contact with the sample). However, our measurements were done under ambient conditions at a high humidity around $50 \%$. At such high humidity, it was not possible to observe any trapped charges induced by either contact charging or triboelectrification. Due to the conductive water layer at a sample surface, the time scale of the relaxation process of the trapped charges is very small (smaller than the time needed to switch to C-AFM (KPFM) mode and make a full scan). At the same time, the relaxation of trapped charges is generally followed by lateral charge spreading. On the other hand, in our case, we didn't observe any spreading of domains with modified surface potential as can be seen in the KPFM images in Fig. 6. In addition, tribocharges generally improve conductivity due to resulting, discharge induced currents, while we observed exactly the opposite behaviour. Therefore, decreased conductivity, the time constant of the relaxation process and clear spatial confinement of domains with the modified electrical surface potential imply that we really induced an internal flexoelectric field, while trapped charges due to triboelectrification can be excluded.

\section{Conclusions}

In summary, we have demonstrated mechanically induced switching of the electrical conductivity of thin manganite films covered by iron-oxide NPs. The sample surface is switched to an insulating state for a high enough local pressure applied by the AFM tip. The pressure induces the flexoelectric field while the corresponding potential barrier prevents further flow of charge carriers from the AFM tip to the sample. After removing the AFM tip, the sample surface is relaxed slowly, with the time constant in the order of hours. During this time, oxygen vacancies diffuse from the bulk of manganite films towards the surface until the equilibrium electrical surface potential is not reached. Top iron-oxide NPs provide weak room-temperature magnetism, protect the surface of manganite films from aging caused by environmental factors, and assure wear resistance and a reliable switching process of manganite films since the NPs are mechanically stable and tightly attached to the underlying manganite films even after the rubbing at high normal loads. The considered heterostructures are potential candidates for novel multifunctional devices with switchable both electric and magnetic properties. In that sense, in order to reach their full potential, the next step would be to explore the switching of their magnetic properties.

\section{Conflicts of interest}

There are no conflicts to declare.

\section{Acknowledgements}

B. V. and Z. K. acknowledge the support of the Serbian Ministry of Education, Science and Technological Development (Projects No. OI171005 and III45018). E. P.-M. and A. P. acknowledge financial support from the Spanish Ministry of Economy and Competitiveness through the "Severo Ochoa" Programme for Centres of Excellence in R\&D (SEV-2015-0496), and project MAT2015-71664 R. This work has received funding from the European Union's Horizon 2020 research and innovation 
programme under the Marie Sklodowska-Curie grant agreement No. 645658 (DAFNEOX Project). B. V. thanks Dimitrije Stepanenko for fruitful discussions.

\section{References}

1 D. B. Strukov, G. S. Snider, D. R. Stewart and R. S. Williams, Nature, 2008, 453, 80-83.

2 J. J. Yang, M. D. Pickett, X. Li, D. A. A. Ohlberg, D. R. Stewart and R. S. Williams, Nat. Nanotechnol., 2008, 3, 429-433.

3 R. Waser and M. Aono, Nat. Mater., 2007, 6, 833-840.

4 A. Sawa, Mater. Today, 2008, 11, 28-36.

5 D. S. Jeong, R. Thomas, R. S. Katiyar, J. F. Scott, H. Kohlstedt, A. Petraru and C. S. Hwang, Rep. Prog. Phys., 2012, 75, 076502.

6 D. B. Strukov and H. Kohlstedt, MRS Bull., 2012, 37, 108-114. 7 D. Ielmini, Semicond. Sci. Technol., 2016, 31, 063002.

8 B. J. Choi, D. S. Jeong, S. K. Kim, C. Rohde, S. Choi, J. H. Oh, H. J. Kim, C. S. Hwang, K. Szot, R. Waser, B. Reichenberg and S. Tiedke, J. Appl. Phys., 2005, 98, 033715.

9 K. Szot, W. Speier, G. Bihlmayer and R. Waser, Nat. Mater., 2006, 5, 312-320.

10 M. Lanza, U. Celano and F. Miao, J. Electroceram., 2017, 39, 94-108.

11 Y. Yang, X. Zhang, L. Qin, Q. Zeng, X. Qiu and R. Huang, Nat. Commun., 2017, 8, 15173.

12 I. Bozovic, G. Logvenov, I. Belca, B. Narimbetov and I. Sveklo, Phys. Rev. Lett., 2002, 89, 107001.

13 J. H. Haeni, P. Irvin, W. Chang, R. Uecker, P. Reiche, Y. L. Li, S. Choudhury, W. Tian, M. E. Hawley, B. Craigo, A. K. Tagantsev, X. Q. Pan, S. K. Streiffer, L. Q. Chen, S. W. Kirchoefer, J. Levy and D. G. Schlom, Nature, 2004, 430, 758.

14 L. Abad, V. Laukhin, S. Valencia, A. Gaup, W. Gudat, L. Balcells and B. Martínez, Adv. Funct. Mater., 2007, 17, 3918-3925.

15 A. Tebano, C. Aruta, S. Sanna, P. G. Medaglia, G. Balestrino, A. A. Sidorenko, R. De Renzi, G. Ghiringhelli, L. Braicovich, V. Bisogni and N. B. Brookes, Phys. Rev. Lett., 2008, 100, 137401.

16 S. Lee, A. Pirogov, M. Kang, K.-H. Jang, M. Yonemura, T. Kamiyama, S.-W. Cheong, F. Gozzo, N. Shin, H. Kimura, Y. Noda and J.-G. Park, Nature, 2008, 451, 805.

17 A. Herklotz, D. Lee, E.-J. Guo, T. L. Meyer, J. R. Petrie and H. N. Lee, J. Phys.: Condens. Matter, 2017, 29, 493001.

18 A. N. Morozovska, E. A. Eliseev, A. K. Tagantsev, S. L. Bravina, L.-Q. Chen and S. V. Kalinin, Phys. Rev. B, 2011, 83, 195313.

19 P. Zubko, G. Catalan and A. K. Tagantsev, Annu. Rev. Mater. Res., 2013, 43, 387-421.

20 P. Zubko, G. Catalan, A. Buckley, P. R. L. Welche and J. F. Scott, Phys. Rev. Lett., 2007, 99, 167601.

21 D. Lee, A. Yoon, S. Y. Jang, J.-G. Yoon, J.-S. Chung, M. Kim, J. F. Scott and T. W. Noh, Phys. Rev. Lett., 2011, 107, 057602.

22 H. Lu, D. J. Kim, C.-W. Bark, S. Ryu, C. B. Eom, E. Y. Tsymbal and A. Gruverman, Nano Lett., 2012, 12, 6289-6292.

23 D. Lee, S. M. Yang, J.-G. Yoon and T. W. Noh, Nano Lett., 2012, 12, 6436-6440.
24 Y. Kim, S. J. Kelly, A. Morozovska, E. K. Rahani, E. Strelcov, E. Eliseev, S. Jesse, M. D. Biegalski, N. Balke, N. Benedek, D. Strukov, J. Aarts, I. Hwang, S. Oh, J. S. Choi, T. Choi, B. H. Park, V. B. Shenoy, P. Maksymovych and S. V. Kalinin, Nano Lett., 2013, 13, 4068-4074.

25 S. J. Kelly, Y. Kim, E. Eliseev, A. Morozovska, S. Jesse, M. D. Biegalski, J. F. Mitchell, H. Zheng, J. Aarts, I. Hwang, S. Oh, J. S. Choi, T. Choi, B. H. Park, S. V. Kalinin and P. Maksymovych, Nanotechnology, 2014, 25, 475302.

26 P. Sharma, S. Ryu, J. D. Burton, T. R. Paudel, C. W. Bark, Z. Huang, Ariando, E. Y. Tsymbal, G. Catalan, C. B. Eom and A. Gruverman, Nano Lett., 2015, 15, 3547-3551.

27 Y. Wang, K. Zhao, X. Shi, G. Li, G. Xie, X. Lai, J. Ni and L. Zhang, Sci. Rep., 2015, 5, 10841.

28 S. Das, B. Wang, Y. Cao, M. Rae Cho, Y. Jae Shin, S. Mo Yang, L. Wang, M. Kim, S. V. Kalinin, L.-Q. Chen and T. W. Noh, Nat. Commun., 2017, 8, 615.

29 A. Gómez, J. M. Vila-Fungueiriño, R. Moalla, G. Saint-Girons, J. Gázquez, M. Varela, R. Bachelet, M. Gich, F. Rivadulla and A. Carretero-Genevrier, Small, 2017, 13, 1701614.

30 G. Catalan, A. Lubk, A. H. G. Vlooswijk, E. Snoeck, C. Magen, A. Janssens, G. Rispens, G. Rijnders, D. H. A. Blank and B. Noheda, Nat. Mater., 2011, 10, 963-967.

31 H. Lu, C.-W. Bark, D. Esque de los Ojos, J. Alcala, C. B. Eom, G. Catalan and A. Gruverman, Science, 2012, 336, 59-61.

32 Y.-J. Li, J.-J. Wang, J.-C. Ye, X.-X. Ke, G.-Y. Gou, Y. Wei, F. Xue, J. Wang, C.-S. Wang, R.-C. Peng, X.-L. Deng, Y. Yang, X.-B. Ren, L.-Q. Chen, C.-W. Nan and J.-X. Zhang, Adv. Funct. Mater., 2015, 25, 3405-3413.

33 Y. Gu, Z. Hong, J. Britson and L.-Q. Chen, Appl. Phys. Lett., 2015, 106, 022904.

34 L. Chen, Z. Cheng, W. Xu, X. Meng, G. Yuan, J. Liu and Z. Liu, Sci. Rep., 2016, 6, 19092.

35 T. Jia, H. Kimura, Z. Cheng and H. Zhao, Sci. Rep., 2016, 6, 31867.

36 Z. Lu, Z. Fan, P. Li, H. Fan, G. Tian, X. Song, Z. Li, L. Zhao, K. Huang, F. Zhang, Z. Zhang, M. Zeng, X. Gao, J. Feng, J. Wan and J. Liu, ACS Appl. Mater. Interfaces, 2016, 8, 23963-23968.

37 D. Guo, G. Xie and J. Luo, J. Phys. D: Appl. Phys., 2014, 47, 013001.

38 K. Yakushiji, F. Ernult, H. Imamura, K. Yamane, S. Mitani, K. Takanashi, S. Takahashi, S. Maekawa and H. Fujimori, Nat. Mater., 2005, 4, 57-61.

39 H. A. Atwater and A. Polman, Nat. Mater., 2010, 9, 205-213. 40 X. Yu, T. J. Marks and A. Facchetti, Nat. Mater., 2016, 15, 383. 41 J.-G. J. Zhu and C. Park, Mater. Today, 2006, 9, 36-45.

42 S. Yuasa, T. Nagahama, A. Fukushima, Y. Suzuki and K. Ando, Nat. Mater., 2004, 3, 868.

43 Z. Xu, M. Gao, L. Yu, L. Lu, X. Xu and Y. Jiang, ACS Appl. Mater. Interfaces, 2014, 6, 17823-17830.

44 Z. Konstantinović, F. Sandiumenge, J. Santiso, L. Balcells and B. Martínez, Nanoscale, 2013, 5, 1001-1008.

45 R. Abrudan, F. Brüssing, R. Salikhov, J. Meermann, I. Radu, H. Ryll, F. Radu and H. Zabel, Rev. Sci. Instrum., 2015, 86, 063902. 
46 E. Dagotto, T. Hotta and A. Moreo, Phys. Rep., 2001, 344, 1153.

47 A.-M. Haghiri-Gosnet and J.-P. Renard, J. Phys. D: Appl. Phys., 2003, 36, R127.

48 T. Wu, S. B. Ogale, J. E. Garrison, B. Nagaraj, A. Biswas, Z. Chen, R. L. Greene, R. Ramesh, T. Venkatesan and A. J. Millis, Phys. Rev. Lett., 2001, 86, 5998-6001.

49 C. Moreno, C. Munuera, S. Valencia, F. Kronast, X. Obradors and C. Ocal, Nano Lett., 2010, 10, 3828-3835.

50 L. Balcells, L. Peña, R. Galceran, A. Pomar, B. Bozzo, Z. Konstantinović, F. Sandiumenge and B. Martínez, J. Appl. Phys., 2013, 113, 073703.

51 A. Tebano, C. Aruta, P. G. Medaglia, F. Tozzi, G. Balestrino, A. A. Sidorenko, G. Allodi, R. De Renzi, G. Ghiringhelli, C. Dallera, L. Braicovich and N. B. Brookes, Phys. Rev. B: Condens. Matter Mater. Phys., 2006, 74, 245116.

52 L. Marín, L. A. Rodríguez, C. Magén, E. Snoeck, R. Arras, I. Lucas, L. Morellón, P. A. Algarabel, J. M. De Teresa and M. R. Ibarra, Nano Lett., 2015, 15, 492-497.

53 J. Ma, Y. Zhang, L. Wu, C. Song, Q. Zhang, J. Zhang, J. Ma and C.-W. Nan, MRS Commun., 2016, 6, 354-359.

54 R. U. Chandrasena, W. Yang, Q. Lei, M. U. Delgado-Jaime, K. D. Wijesekara, M. Golalikhani, B. A. Davidson, E. Arenholz, K. Kobayashi, M. Kobata, F. M. F. de Groot,
U. Aschauer, N. A. Spaldin, X. Xi and A. X. Gray, Nano Lett., 2017, 17, 794-799.

55 Z. Konstantinović, J. Santiso, L. Balcells and B. Martínez, Small, 2009, 5, 265-271.

56 Z. Konstantinović, J. Santiso, D. Colson, A. Forget, L. Balcells and B. Martínez, J. Appl. Phys., 2009, 105, 063919.

57 Z. Konstantinović, L. Balcells and B. Martínez, J. Magn. Magn. Mater., 2010, 322, 1205-1208.

58 A. Tebano, C. Aruta, P. G. Medaglia, F. Tozzi, G. Balestrino, A. A. Sidorenko, G. Allodi, R. De Renzi, G. Ghiringhelli, C. Dallera, L. Braicovich and N. B. Brookes, Phys. Rev. B: Condens. Matter Mater. Phys., 2006, 74, 245116.

59 L. Yao, S. Inkinen and S. van Dijken, Nat. Commun., 2017, 8, 14544.

60 Y.-L. Chung, P. Y. Lai, Y.-C. Chen and J.-S. Chen, ACS Appl. Mater. Interfaces, 2011, 3, 1918-1924.

61 J. M. D. Coey, M. Viret and S. von Molnár, Adv. Phys., 2009, 58, 571-697.

62 M. Andra, F. Gunkel, C. Baumer, C. Xu, R. Dittmann and R. Waser, Nanoscale, 2015, 7, 14351-14357.

63 T. Bieger, J. Maier and R. Waser, Sens. Actuators, B, 1992, 7, 763-768.

64 R. Merkle and J. Maier, Angew. Chem., Int. Ed., 2008, 47, 3874-3894. 